\title{
Evolution towards a Cloud Deployed Business Support System
}

\author{
Ioan DRĂGAN, Răzvan Daniel ZOTA \\ Bucharest University of Economic Studies \\ dragan_ioan1989@yahoo.com, zota@ase.ro
}

Although less known outside strictly specialized environments, Business Support Systems (BSS) are highly complex and the subject of their installation in cloud implementations is less addressed. This paper presents a short history of BSS evolution, starting from basic voice and messaging services and ending up to $4 G$ and M2M services, presenting new features and their new challenges. Moreover, we present, as a baseline for future developments, a study based on direct interviews with representatives of telecom operators about their vision of possible future BSS solutions depending on the services they will provide. This area of investigation has a certain number of challenges that require collaboration between providers and operators; in this context, we have been established a framework of requirements which will be handled and studied individually.

Keywords: Business Support Systems, Cloud implementations, Telecom Providers

1 Introduction

In one of the simplest forms, business support systems (BSS) represent the "connection point" between external relations (customers, suppliers and partners) and an enterprise's products and services. Moreover, products and services are correlated with corresponding resources, like networking infrastructure, applications, contents and factories [1].

Translating into a more comprehensive language, Business Support Subsystems is an aggregation of functions that are used for managing an operator's day-to-day business and providing an operator with complete picture on the performance and management of his diverse lines of businesses.

Basically, a BSS has to handle the taking of orders, payment issues, revenues and managing customers, etc. According to eTOM Framework it supports four processes: product management, order management, revenue management and customer management [2].

- Product management supports product development, sales and management of products, offers and bundles addressed to businesses and regular customers. Product management regularly includes offering product discounts, appropriate pricing and managing how products relate to one another.

- Customer management. Service providers require a single view of the customer and need to support complex hierarchies across customer-facing applications also known as customer relationship management. Customer management also covers the partner management and $24 \times 7$ web-based customer self-service.

- Revenue management is focused on billing, charging and settlement.

- Order management involves taking and handling the customer order. It encompasses four areas: order decomposition, order orchestration, order fallout and order status management. 


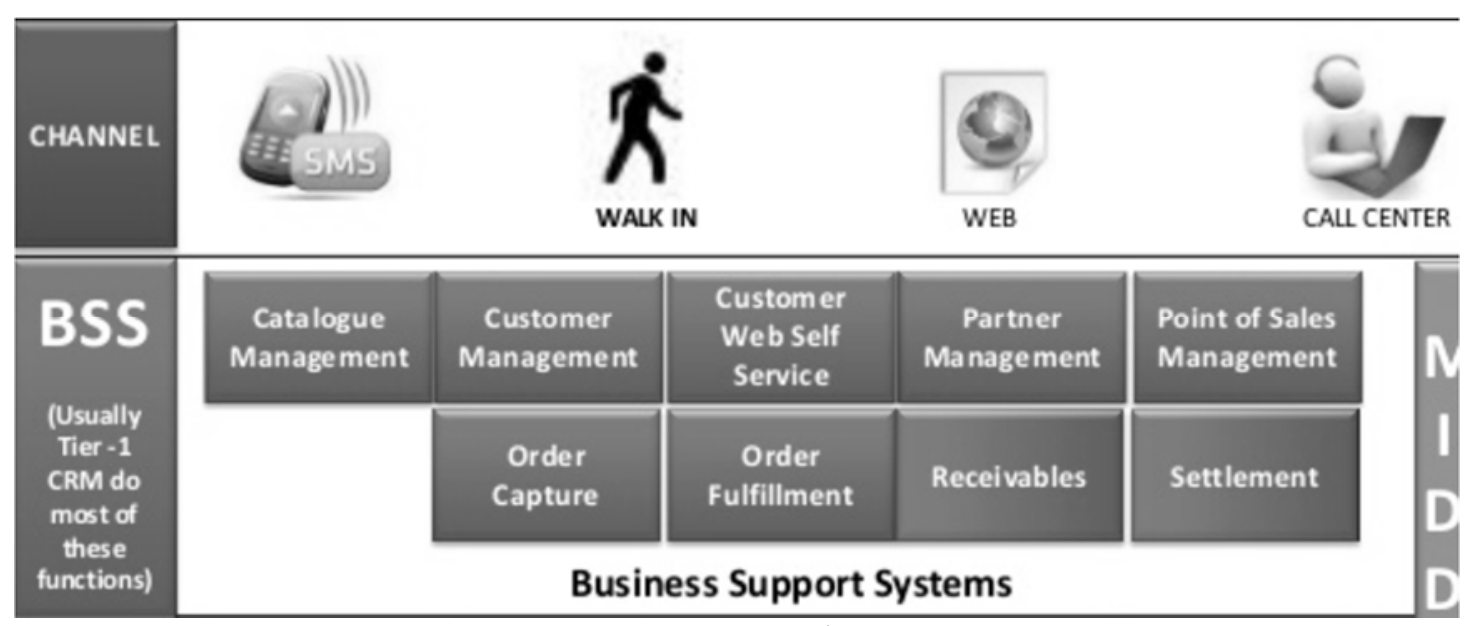

Fig. 1. BSS Functions [3]

In order to identify the main characteristics several market research methods have been used:

- Research of existing BSS software providers and analysis of their top selling products

- Interviews with telecom operators' representatives

- Other existing studies based on market available products

- eTOM standard [3]

By following these methods, some quantitative and qualitative key performance indicators were applied that would help us understand the operators' needs and how we can develop this as a collaborative system [4]:

- Qualitative: user experience, ability to adopt new services, operator on boarding experience, interconnection between on premise equipment and cloud based software, quality of this interoperation and coordination;

- Quantitative: number of customers it can support, number of operators it can support, handling processing peeks and data retention policy.

\section{Traditional BSS Services}

Traditional telecom networks made money by providing technology to connect users and services that are derived from that technology. To achieve optimum return on investment, network equipment and services investments were made with a very long life cycle. The products' expectation to be in ser- vice was from five up to 20 years, but technology evolution proved them wrong.

Initially, BSS systems supported a limited number of services:

- Customer relationship management - this was used by telecom operators to manage their customers, mostly based on human intervention from qualified personnel

- Billing services - based on service usage the customers were billed and an invoice was sent to them monthly.

- Order handling - orders were mostly handled manually by operators; this was only a matter of registering and tracking them.

Services supported by traditional BSS solutions:

- Voice calls - initially, voice calls were billed based on connection time, without any reference to source or destination of the call

- SMS - billed based on number of text messages, without any source/destination considerations

- Data - In 1991, 2G was available and provided also data services, which was a challenge to be billed. Initially, data services were billed based on the connection minutes

Billing services evolved into complicated price plans based on source, destination, roaming, time based triggers and other constrains, but was still based on strict usage and monthly fees. It did not provide any means of charging other experience-based services. 
Customer management systems evolved into self-service portals up to some degree, but still do not offer any means of dynamically assigning services based on customer profile [5].

The next generation BSS is considered to be linked to the launch of $3 \mathrm{G}$, which provided high-speed internet connections and add on services based on the new service layer.

\section{Evolution towards Multi System Inte- grated Services}

\subsection{Service Evolution}

The introduction of 4G/LTE networks created a multitude of new opportunities for wireless operators but also presented a set of new challenges.

\section{OSS \& BSS: evolution history}

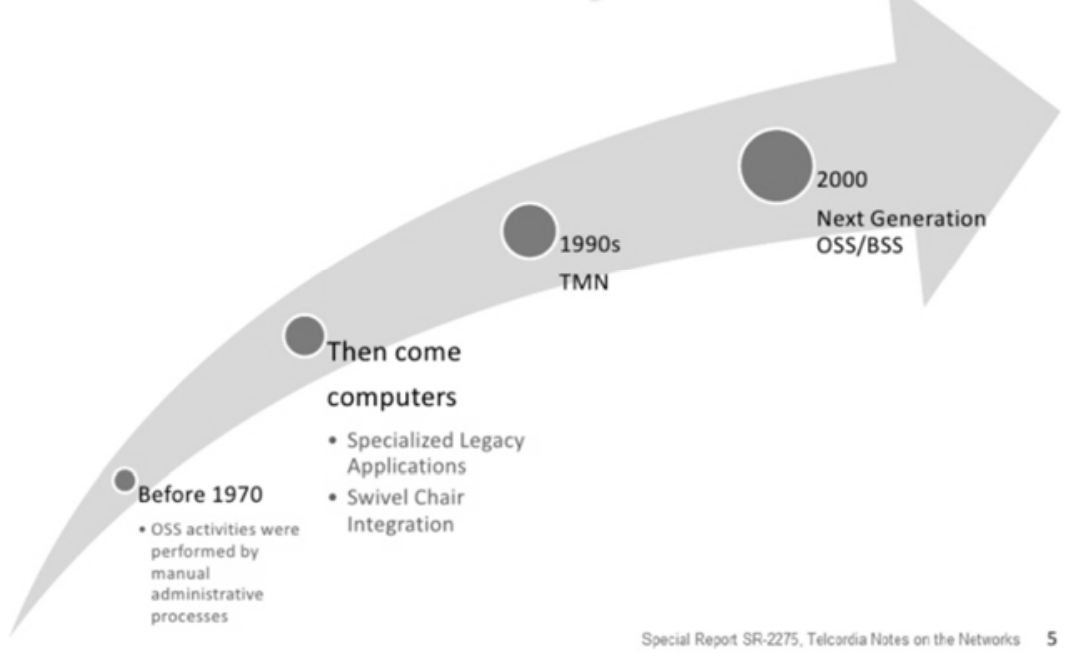

Fig. 2. BSS evolution [6]

It's giving telecom operators the chance to develop new, differentiated wireless services, and potentially new sources of revenue like [4]:

- Content distribution: audio/video

- Software services for enterprises. E.g. mobile device management, subscription based office solutions

- Mobile payment solutions

According to telecom operators, the top telecom industry trends in BSS systems and architecture are being driven by service layer architecture and the need to manage customer experiences rather than subscription services. The major changes include the following:

- Transforming from a supply to demand side vision of the business:

For network operators to be successful, they must adapt their offering to many different customers in the marketplace, ranging from regular users, to high data consuming services and even M2M (machine to machine). These circumstances push the operators to limit the gap between operations support system and the business support system. It has to be ready to adapt to any new business model that proves to be efficient, even though requirements might not be clearly understood from the beginning. Overall, the new business vision has to [7]:

- Create a relevant experience for the users and provide a wide range of products, services and even tailored services;

- Empower collaboration with external services providers in order to monetize and share revenue with them;

- Finally yet importantly, provide a unified support and simple admin interface for M2M opportunities. 


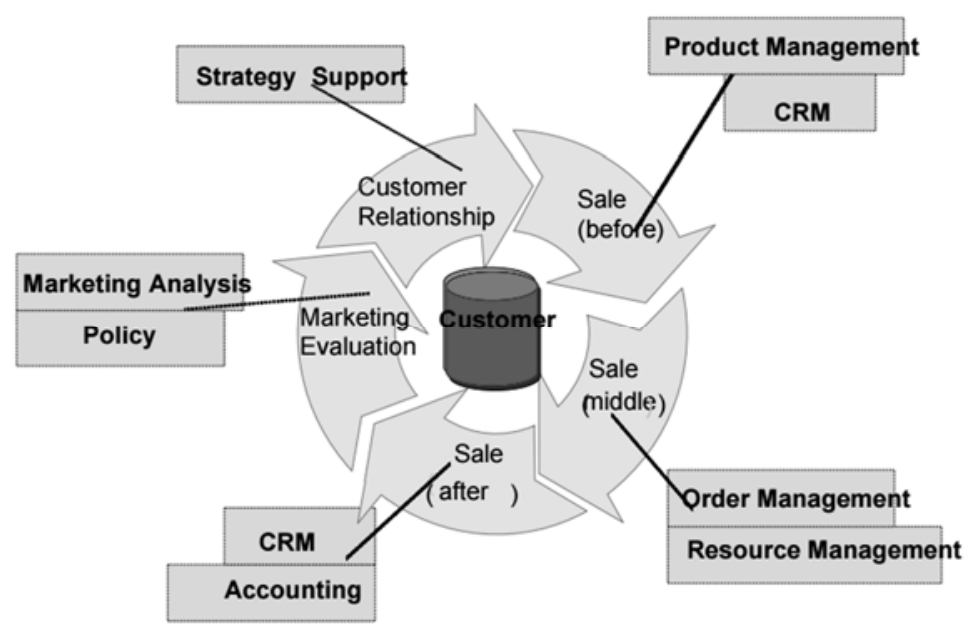

Fig. 3. Telecom Business Process

- Transforming from human personnel support to automated support

Human personnel support is usually slow and does not offer the expected user experience for enterprise services.

Even though automated customer service can get a bad reputation, it has proven that, addressing to the right audience can save a lot of employee time and money and put back personnel into personal services.

The new business support systems have to be integrated with the automated support solutions in order to provide change/self-service actions and integrate it with a feedback and machine learning system. Customers' needs change and there is no better way of understanding them.

Increased competition and the demand for instant service consumption, diverse niches of subscribers drives operators to change towards reinventing their support. Lately we have noticed an evolution from all manual support towards interactive voice responses, interactive support menus and even onlineautomated support areas.

- Transforming from "management as an overlay" to "management as service logic".

In the telecom operators' network of the future vision, the "services" will involve the experiences from a dynamic composition of transport/connection resources, content and processing resources, subscriber knowledge based on customer's behaviour and location. This new vision is also reflected in the transformation of the eTOM through the addition of processes support like the Service Delivery Framework (SDF), the Telecommunications Interface Program (TIP) and the Integration Framework.

These will provide a fresh new dimension to the older tom processes and link long-cycle services and products planning with shortercycle, software-driven service creation. Over the next four years, these new views of BSS systems will dominate and eventually replace the old static product based BSS systems.

The future services will be created through software processes and aimed at supporting experiences instead of customers and will be more complex and much more numerous than static products of the past.

New methods of handling this are being developed because operations costs cannot be allowed to scale proportionally to the number of services or to grow exponentially based on the number of component relationships. That means that the BSS systems of the past, which supported human-driven provisioning processes, must support automated and included in a self-service package with software-based dynamic services. 


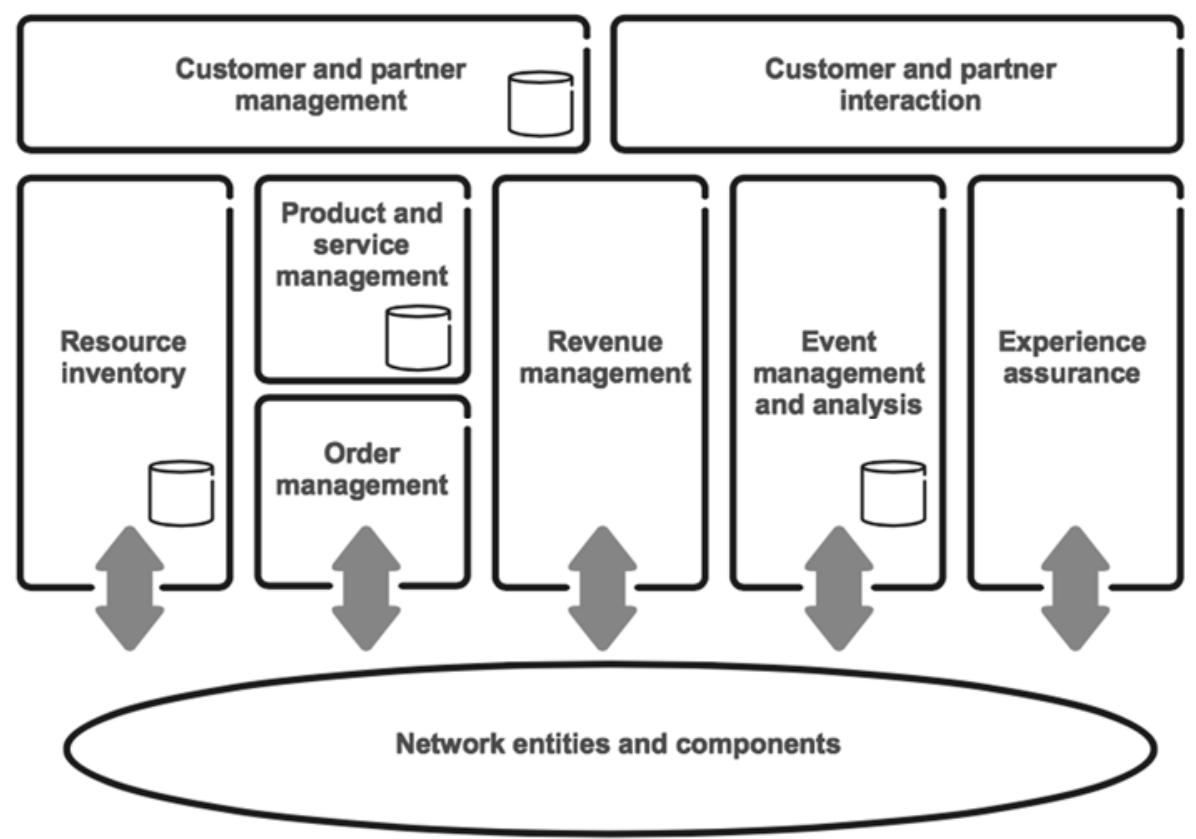

Fig. 4. New BSS model [8]

Traditionally, the operators offered mainly messaging and payment services. In the new market landscape, they have to provide at the click of a button subscriber information, network quality of service and also other business processes related to self-service, order management and fulfilment.

Subscribers perceive the value of their experience based on how they access the services included in the subscription package, whether that is messaging, data connectivity, streamed applications or content. They also seek a unified, yet customized experience of their services, across devices. However, traditional BSS systems were not designed for providing these experience-driven service level agreements, user experience key performance indicators or dynamic personalization capabilities.

Considering the wide range of services that have to be handled by the new BSS, an increasing number of user roles have to be implemented: company administrators, user management administrators, billing and payment administrators, service administrators etc. Each of this user role has to receive a personalized experience in order to properly perform his role. [9]

The forecasts that predicts 50 billion mobile connected devices by 2017 are staggering.
Especially that, when you consider these forecasts are based only upon devices like smart phones, smart tablets, smart meters and any other M2M controllers - whereas the connectivity itself is central to the device's function. [10]

\subsection{Network Evolution Directly Impacting BSS}

\subsubsection{IMS}

A parallel evolution of the classic services is the IMS (IP Multimedia Subsystem). The IMS is a new concept for an integrated network of telecom carriers that would publish all the existing services, wireless or landline, on an IP network, rather than individual services. Examples of such services would be: voice, video conferencing, video on demand, instant messaging etc.

Having an IMS architecture in place complicates even more then BSS architecture, since it has to avoid double charging the customer per service and per data consumption.

TMF is currently working to adapt the eTOM architecture to support a service-oriented architecture. However, eTOM remains biased on the inheritance of process management for new services rather than building the service itself. [11] 
To overcome the technological challenges posed by IMS implementation and to exploit the full potential of IMS services, telecom operators require a BSS architecture and systems that support the three main points of service excellence: rapid time-to-market, flexibility and reduced operating expenses.

\subsubsection{G}

The introduction of $4 \mathrm{G}$ networks creates new opportunities for telecom operators to increase their revenue and expand their portfolio. Creating new services comes with a fresh new set of challenges, mainly related to managing, billing and combining the services into easy to use packages.

The telecom market becomes highly competitive since the operators are competing for a finite number of clients with low possibility of expanding the market.

The 4G era is strictly tied to the IMS evolution, since 4G networks do not support Circuit Switched services anymore (voice, traditional video calls etc.). So mainly all the services that we know have to be emulated on IP networks in order to be compatible to $4 \mathrm{G}$ networks.

The foundation of an effective BSS system in the context of $4 \mathrm{G}$ networks will rely on the following fundamental requirements [13]:

- A centralized product catalogue

- Effective network policy management integrated with the OSS system

- BSS systems should be able to combine customer usage and subscription data with insight into the network

- BSS system in 4G context should be able to provide prepaid and post-paid charging on the same platform

- Embedded intelligence and real-time analytics based decision

- BSS solutions for $4 \mathrm{G}$ need to support the full product lifecycle, from creation of products to post sales activities.

\section{Cloud Deployment Study}

In the study performed on telecom operators some common concerns have been raised regarding the deployment of BSS solutions on cloud environments:
- Multitude of BSS products providers and usually no simple migration means from one to another.

This has been raised by telecom operators who were forced to migrate from one vendor to another and had no options available for a simple migration. Since service providers that offer cloud solutions will choose at best an existing vendor or most probably develop a new product suitable for this kind of deployment, migrating data from on premise solutions will be a challenge from technical point of view and also a matter of costs which has to be covered. Currently there is no available BSS cloud service that offers data migration.

Transferring data between computer systems or storage systems is never an easy task. Most computer systems have a mix between structured and unstructured data formats that have to be translated into the new business models that the target software provides.

During interviews, telecom operators mentioned that backwards compatibility is always a requirement for upgrading their BSS. Mainly, they use the same vendors for each of their components to avoid having to redesign their data model. Having to move to a brand new product, adds a number of extra concerns related to data integrity after migration and business functionalities backwards compatibility.

Running through the interviews, other common concerns were identified that have to be treated with high priority:

1 Legacy systems poor data quality

2 Very large amounts of data in legacy systems backup of historical data that has to be migrated or at least kept in a human readable format

3 Network bandwidth consumed to move this data

4 No means of validating migrated data quality against the legacy systems

5 No industry standard data model is present

In order to capture also their previous experience with this kind of data model, we have attempted to identify their previous failures in migrating data from one system to another 
and quantify them into risks, costs of mitigation or fixing and probability of happening:

1 IT resources are not business process experts. Fail to involve all the business owners will result in data migrated without their underlying business follows. The cost of fixing this event is very high, considering that business interruptions might occur. The probability of happening is medium.

2 Bad data is migrated. The cost of mitigation is usually low, consisting only in manual corrections, but the risk of happening is very high.

3 Data is migrated in one big batch before the rollout and it is not consistent with the business needs. This bad practice is usually avoided in business critical systems, but the cost of fixing this is not very high, if properly mitigated from the beginning.

4 Budget constraints that are usually overran, due to inadequate scoping. The probability of happening is very high, but usually budgeted from the beginning.

- No carrier grade cloud BSS services available on the market.

Existing cloud BSS services offer at most $99.9 \%$ availability and scalability up to a medium MVNO(Mobile Virtual Network Operator), but none of them offers a carrier grade service availability $99.999 \%$ ("the five $9 \mathrm{~s}$ " as it is called by telecom operators) with scalability up to tens of millions of subscribers. Since this is a "niche" market, such products were not suitable until now from costs perspective. The only "promise of delivery" is Ericsson's "BSS as a service" which should be available at the beginning of 2016. This will be based on Ericsson products only and might offer some degree of compatibility with on premise deployments.

Interviewed operators have raised concerns about availability criteria that are offered currently on the market. Considering the fact that some of their services are business critical and can have no interruption, network availability and cloud service availability has to be considered.
As probably known by industry specialist, availability is calculated by multiplying each of its components individual availability. Having one of them failing will results in a failure of the whole system.

By calculating the $99.999 \%$ SLA unplanned downtime window it results in 5.2 minutes per year, cumulated. Searching among current solutions, the highest SLA available on the market is $99.9 \%$, which is not remotely close to their needs.

Many people also confuse two key terms in measuring a software system's quality: reliability and availability. This to terms, even though linked, are totally independent and should be considered separately.

Reliability is a measure of likelihood that a system will be $100 \%$ available for a specific period of time. This should be associated with the mean time before failure and the probability that it will reach some of this targets.

Availability on the other hand, is a relative measure of the extent that a system can perform it's designated functions.

So the $99.999 \%$ should be calculated off system uptime and also system's ability to perform the designated tasks at the expected speed and quality.

This will make a subject for a future research to establish a set of KPIs that have to be fulfilled in order to have a continuously available and continuously functioning system.

- No options to pay for occasional extension of capacity and changing of SLA/QoS (ex. monthly bill run, New Year's eve, other planned events).

Current pay as you grow subscriptions (or pay for what you use) allow limited capacity extension and require complex setups in some occasions. Telecom operators currently use "ready to deploy" virtual machines for capacity expansions in case of special events like: monthly bill run, New Year's Eve, discounts or historical bills adjustments. Meanwhile, these VMs are turned off and this capacity is used for other activities. Deploying and configuring VMs every time it's needed on public/shared cloud infrastructure would 
add extra complexity and human intervention. Currently there are no available software solutions for "event aware" selfdefining infrastructure.

"Pay As You Go (PAYG) is a utility computing billing method that is implemented in cloud computing and geared toward organizations and end users. A PAYG user is billed for procured, rather than actual, computing resources. The PAYG mechanism is derived from utility computing." [12]

As stated above in the definition of Pay As You GO services, the operators has to pay only for computational resources that he used, but there is no mention of dynamically changing the SLA.

As stated in the interviews, telecom operators require for some business components different QoS over a period of time. As stated in the above paragraph, high availability is expensive, some requirements not even yet available on the market. Purchasing a service with $99.999 \%$ availability for the whole period of time could exceed a few times fold the budget. This kind of availability is needed only during peak hours. Adapting the SLA to the business requirements in order to reduce costs is a critical requirement that currently is not available on the market.

\section{Conclusions}

Nowadays, telecom operators are looking for new sources of revenue based on customer experience rather than fix priced products. New business models based on revenue sharing are now used in partnerships between telecom operators and software vendors. Customers are now getting software for free and paying only for usage/data plans/data consumptions.

The introduction of 4G/LTE networks and high speed Wi-Fi hotspots allowed telecom operators to distribute media content like music or videos. Even though, this media content is distributed as free of charge, the data consumption is charged accordingly.

This multitude of services has to be managed by the new BSS model. Since there are numerous combinations of services, human operators cannot handle them on a regular ba- sis. Based on the customer's profile, services have to be offered as an add-on experience or via a self-service approach. Integrating with other software or media vendors is a must and cloud deployments would enable a separation of concerns: BSS solution suppliers would handle the enterprise integration and telecom operators would develop new experiences for their customers.

Eventually, cloud deployments present numerous problems since this niche software did not present a financial interest for cloud solutions provides. Solving these problems is actually a matter of processes and convincing telecom operators to invest time and effort and work together with their software or media partners and cloud service providers.

In order to comply with the strict requirements of telecom operators, we have firstly to understand their needs, even challenge that are not backed up by business requirements and fill in the gaps in cloud offerings.

Technical requirements for cloud infrastructure are being covered by service evolution as we speak, so our main concerns should be business processes and business requirements that have to be covered with a BSS solution "one size fits all".

Future research will be directed towards identifying the most suitable components to accommodate telecom operator's requests and developing a proof of concept cloud deployment. This will be presented to the same audience in order to study it and provide the following feedback:

- Qualitative analysis of the system: does it fit your current needs? How would you improve it? Does it provide the expected processing speed? Can it accommodate your new services?

- Quantitative analysis of the system: does it scale enough to your needs? Can it accommodate more than one operator?

\section{Acknowledgment}

Part of the present work is done under the auspices of the doctoral program in Economic informatics - the doctoral school of $\mathrm{Bu}$ charest University of Economic Studies. 


\section{References}

[1] L. Angelin, U. Ollson, P. Tengroth. Business Support Systems. Internet: http://www.ericsson.com/res/thecompany /docs/publications/ericsson_review/2010/ business_support_systems.pdf $\quad[\mathrm{Feb}$, 2010].

[2] eTOM - The Business Process Framework, pages 41-49, GB921B [Mar, 2014]

[3] Tony Poulos. The BSS/OSS Best. TMforum. Internet: http://www.tmforum.org/ArticleTheBSS OSS/9835/home.html [Dec, 2010]

[4] C. Ciurea, "A Metrics Approach for Collaborative Systems", Informatica Economica vol.13, no. 2/2009

[5] "Integrated platform for financial transactions and electronic banking services made available on mobile devices using the technology with widespread" - SERAFIMO, Contract PN II nr. 3039/01.10.2008

[6] Evolution of OSSBSS - Telcordia workshop, Ericsson Review [Dec, 2013] [Online].

Available: http://www.slideshare.net/Ericsson/nextgeneration-ossbss-architecture

[7] Ericsson AB, whitepaper, "OSS/BSS evolution for the new market landscape", http://www.ericsson.com/res/docs/whitep apers/wp-oss-bss-evolution.pdf [September, 2013]

[8] "Ericsson BSS vision" in Mobile World Congress, Barcelona, [Mar, 2013].

[9] Ericsson AB, whitepaper, "OSS/BSS evolution for the new market landscape", http://www.ericsson.com/res/docs/whitep apers/wp-oss-bss-evolution.pdf [September, 2013]

[10] CSGI, whitepaper, "Profiting from the Internet of Things: Above and Beyond Connectivity" [Jan, 2013]

[11]International Engineering Consortium, "Business Models and Drivers for NextGeneration IMS Services", 2007

[12]

Technopedia, http://www.technopedia.com

[13] Ericcson AB, "Next Generation BSS/OSS Architecture", ISSN 00140171, November 2013

[14] Lan Wang and Tingjie Lv, "The NGOSS Evolution of Telecom Service Providers: From Network-Focused to Customers-Focused", School of Economics and Management, Beijing University of Posts and Telecommunications, February 2007

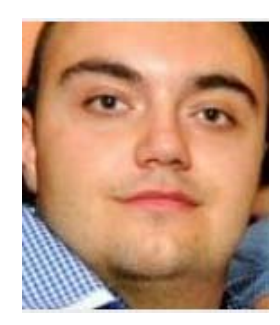

Ioan DRAGAN has graduated the Faculty of Cybernetics, Statistics and Economic Informatics in 2011 and IT\&C Security Master in 2013. Currently he is a PhD candidate at Economic Informatics ASE Bucharest.

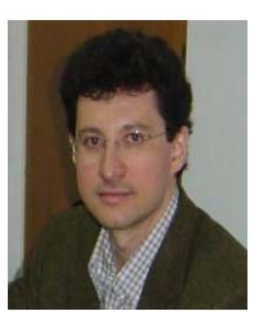

Răzvan Daniel ZOTA has graduated the Faculty of Mathematics Informatics at the University of Bucharest in 1992. He holds a Ph.D. in Economic Informatics from 2000 and now is professor at the Department of Economic Informatics and Cybernetics from the Bucharest University of Economic Studies. From 2010 he is PhD supervisor in the field of Economic Informatics. His last published books in 2004 are "IT Basics" and "Computer Networks" in ASE Publishing House, Bucharest, Romania. His recent work focuses on business cloud computing, computer networks and applications. 